\title{
Weakly Nonlocal Hamiltonian Structures: Lie Derivative and Compatibility ${ }^{\star}$
}

\author{
Artur SERGYEYEV
}

Mathematical Institute, Silesian University in Opava, Na Rybnícku 1, 74601 Opava, Czech Republic

E-mail: Artur.Sergyeyev@math.slu.cz

Received December 15, 2006, in final form April 23, 2007; Published online April 26, 2007

Original article is available at http://www.emis.de/journals/SIGMA/2007/062/

\begin{abstract}
We show that under certain technical assumptions any weakly nonlocal Hamiltonian structure compatible with a given nondegenerate weakly nonlocal symplectic structure $J$ can be written as the Lie derivative of $J^{-1}$ along a suitably chosen nonlocal vector field. Moreover, we present a new description for local Hamiltonian structures of arbitrary order compatible with a given nondegenerate local Hamiltonian structure of zero or first order, including Hamiltonian operators of the Dubrovin-Novikov type.
\end{abstract}

Key words: weakly nonlocal Hamiltonian structure; symplectic structure; Lie derivative

2000 Mathematics Subject Classification: 37K10; 37K05

\section{Introduction}

Nonlinear integrable systems usually are bihamiltonian, i.e., possess two compatible Hamiltonian structures. This ingenious discovery of Magri [14] has naturally lead to an intense study of pairs of compatible Hamiltonian structures both in finitely and infinitely many dimensions, see e.g. $[1,3,5,13,16,24,26,30]$ and references therein.

Using the ideas from the Lichnerowicz-Poisson cohomology theory [13, 30] it can be shown $[5,26]$ that under certain minor technical assumptions all Hamiltonian structures compatible with a given nondegenerate Hamiltonian structure $P$ can be written as the Lie derivatives of $P$ along suitably chosen vector fields. This allows for a considerable reduction in the number of unknown functions: roughly speaking, we deal with components of a vector field rather than with those of a skew-symmetric tensor, and the number of the former is typically much smaller than that of the latter, see e.g. [26] for more details. This idea works well for compatible pairs of finite-dimensional Hamiltonian structures [26, 28] and of local Hamiltonian operators of Dubrovin-Novikov type [21, 26], when the corresponding vector fields are local as well.

In the present work we extend this approach to the weakly nonlocal [16] Hamiltonian structures using weakly nonlocal vector fields. To this end we first generalize the local homotopy formula (7) to weakly nonlocal symplectic structures in Theorem 2 below. This enables us to characterize large classes of Hamiltonian structures compatible with a given weakly nonlocal symplectic structure using the weakly nonlocal (co)vector fields, i.e., elements of $\tilde{\mathcal{V}}$ (resp. $\tilde{\mathcal{V}}^{*}$ ), as presented below in Theorems 3 and 4 and Corollaries 2, 3, 4, and 5 .

The paper is organized as follows. In Section 2 we recall some basic features of infinitedimensional Hamiltonian formalism. Section 3 contains the main theoretical results of the paper while Sections 4 and 5 deal with the particular cases of local Hamiltonian structures of zero and first order where important simplifications occur. Finally, in Section 6 we briefly discuss the results of the present work.

${ }^{\star}$ This paper is a contribution to the Vadim Kuznetsov Memorial Issue 'Integrable Systems and Related Topics'. The full collection is available at http://www.emis.de/journals/SIGMA/kuznetsov.html 


\section{Preliminaries}

Following [5, 24], recall some basic aspects of infinite-dimensional Hamiltonian formalism for the case of one independent variable $x \in B$ (usually $B=\mathbb{R}$ or $B=S^{1}$ ) and $n$ dependent variables.

We start with an algebra $\mathcal{A}_{j}$ of smooth functions of $x, \boldsymbol{u}, \boldsymbol{u}_{1}, \ldots, \boldsymbol{u}_{j}$, where $\boldsymbol{u}_{k}=\left(u_{k}^{1}, \ldots, u_{k}^{n}\right)^{T}$ for $k>0$ are $n$-component vectors from $\mathbb{R}^{n}, \boldsymbol{u}_{0} \equiv \boldsymbol{u} \in M \subset \mathbb{R}^{n}, M$ is an open domain in $\mathbb{R}^{n}$, and the superscript $T$ indicates the transposed matrix. Set $\mathcal{A}=\bigcup_{j=0}^{\infty} \mathcal{A}_{j}$. The elements of $\mathcal{A}$ are called local functions.

Consider (see e.g. [5] and [24] and references therein) a derivation of $\mathcal{A}$

$$
D \equiv D_{x}=\partial / \partial x+\sum_{j=0}^{\infty} \boldsymbol{u}_{j+1} \partial / \partial \boldsymbol{u}_{j}
$$

and let $\operatorname{Im} D$ be the image of $D$ in $\mathcal{A}$, and $\overline{\mathcal{A}}=\mathcal{A} / \operatorname{Im} D$. The space $\overline{\mathcal{A}}$ is the counterpart the algebra of (smooth) functions on a finite-dimensional manifold in the standard de Rham complex. Informally, $x$ can be thought of as a space variable and $D$ as a total $x$-derivative, cf. e.g. [24].

The canonical projection $\pi: \mathcal{A} \rightarrow \overline{\mathcal{A}}$ is traditionally denoted by $\int d x$, and for any $f, g \in \mathcal{A}$ we have

$$
\int f D(g) d x=-\int g D(f) d x .
$$

The quantity $\mathcal{F}=\int f d x$ should not be confused with a nonlocal variable $D^{-1}(f)$ : these are different objects. Informally, $\int f d x$ can be thought of as $\int_{B} f d x$, i.e., this is, roughly speaking, a definite $x$-integral, and $D^{-1}(f)$ is a formal indefinite $x$-integral. If $f \notin \operatorname{Im} D$ then $D^{-1}(f) \notin \mathcal{A}$, and we need to augment $\mathcal{A}$ to include a nonlocal variable $\omega$ such that $D(\omega)=f$ and to extend the action of $D$ accordingly, see below for further details.

The generalized Leibniz rule [17, 18, 19, 24]

$$
a D^{i} \circ b D^{j}=a \sum_{q=0}^{\infty} \frac{i(i-1) \cdots(i-q+1)}{q !} D^{q}(b) D^{i+j-q}
$$

turns the space $\operatorname{Mat}_{q}(\mathcal{A}) \llbracket D^{-1} \rrbracket$ of formal series in powers of $D$ of the form $L=\sum_{j=-\infty}^{k} h_{j} D^{j}$, where $h_{j}$ are $q \times q$ matrices with entries from $\mathcal{A}$, into an algebra, and the commutator $[P, Q]=$ $P \circ Q-Q \circ P$ further makes $\operatorname{Mat}_{q}(\mathcal{A}) \llbracket D^{-1} \rrbracket$ into a Lie algebra. In what follows we shall often omit the composition sign $\circ$ (for instance, we shall write $K L$ instead of $K \circ L$ ) wherever this does not lead to a possible confusion.

The degree $\operatorname{deg} L$ of formal series $L=\sum_{j=-\infty}^{k} h_{j} D^{j} \in \operatorname{Mat}_{q}(\mathcal{A}) \llbracket D^{-1} \rrbracket$ is $[17,18,19,24]$ the greatest integer $m$ such that $h_{m} \neq 0$. If, moreover, det $h_{m} \neq 0$ we shall call $L$ nondegenerate, and then there exists a unique formal series $L^{-1} \in \operatorname{Mat}_{q}(\mathcal{A}) \llbracket D^{-1} \rrbracket$ such that $L^{-1} \circ L=L \circ L^{-1}=\mathbb{I}_{q}$, where $\mathbb{I}_{q}$ stands for the $q \times q$ unit matrix. For any $L=\sum_{j=-\infty}^{m} h_{j} D^{j} \in \operatorname{Mat}_{q}(\mathcal{A}) \llbracket D^{-1} \rrbracket$ let $L_{+}=\sum_{j=0}^{m} h_{j} D^{j}$ denote its differential part, $L_{-}=\sum_{j=-\infty}^{-1} h_{j} D^{j}$ its nonlocal part (so $\left.L_{-}+L_{+}=L\right)$, and let $L^{\dagger}=\sum_{j=-\infty}^{m}(-D)^{j} \circ h_{j}^{T}$ stand for the formal adjoint of $L$, see e.g. $[17,18,19,24]$. A formal series $L$ is said to be skew-symmetric if $L^{\dagger}=-L$. As usual, an $L \in \operatorname{Mat}_{q}(\mathcal{A}) \llbracket D^{-1} \rrbracket$ is said to be a purely differential (or just differential) operator if $L_{-}=0$.

Let $\mathcal{A}^{q}$ be the space of $q$-component functions with entries from $\mathcal{A}$, no matter whether they are interpreted as column or row vectors. For any $\vec{f} \in \mathcal{A}^{q}$ define (see e.g. [12]) its directional derivative as

$$
\overrightarrow{f^{\prime}}=\sum_{i=0}^{\infty} \partial \vec{f} / \partial \boldsymbol{u}_{i} D^{i}
$$


We shall also need the operator of variational derivative (see e.g. $[1,5,24,2]$ )

$$
\delta / \delta \boldsymbol{u}=\sum_{j=0}^{\infty}(-D)^{j} \circ \partial / \partial \boldsymbol{u}_{j} .
$$

Following [16], an $L \in \operatorname{Mat}_{q}(\mathcal{A}) \llbracket D^{-1} \rrbracket$ is called weakly nonlocal if there exist $\vec{f}_{\alpha} \in \mathcal{A}^{q}, \vec{g}_{\alpha} \in \mathcal{A}^{q}$ and $k \in \mathbb{N}$ such that $L_{-}=\sum_{\alpha=1}^{k} \vec{f}_{\alpha} \otimes D^{-1} \circ \vec{g}_{\alpha}$. Nearly all known today Hamiltonian and symplectic operators in $(1+1)$ dimensions are weakly nonlocal, cf. e.g. [32]. Recall that an operator of the form $L=\vec{f} \otimes D^{-1} \circ \vec{g}$ acts on an $\vec{h} \in \mathcal{A}^{q}$ as follows:

$$
L(\vec{h})=\left(D^{-1}(\vec{g} \cdot \vec{h})\right) \vec{f}
$$

where "." denotes the standard Euclidean scalar product in $\mathcal{A}^{q}$.

Denote by $\mathcal{V}$ the space of $n$-component columns with entries from $\mathcal{A}$. The commutator $[\boldsymbol{P}, \boldsymbol{Q}]=\boldsymbol{Q}^{\prime}[\boldsymbol{P}]-\boldsymbol{P}^{\prime}[\boldsymbol{Q}]$ turns $\mathcal{V}$ into a Lie algebra, see e.g. [1, 12, 18, 24]. The Lie derivative of $\boldsymbol{R} \in \mathcal{V}$ along $\boldsymbol{Q} \in \mathcal{V}$ reads $L_{\boldsymbol{Q}}(\boldsymbol{R})=[\boldsymbol{Q}, \boldsymbol{R}]$, see e.g. [1, 5, 31, 24]. The natural dual of $\mathcal{V}$ is the space $\mathcal{V}^{*}$ of $n$-component rows with entries from $\mathcal{A}$.

The canonical pairing of $\mathcal{V}$ and $\mathcal{V}^{*}$ is given by the formula (see e.g. [5, 32])

$$
\langle\gamma, \boldsymbol{Q}\rangle=\int(\boldsymbol{\gamma} \cdot \boldsymbol{Q}) d x
$$

where $\gamma \in \mathcal{V}^{*}, \boldsymbol{Q} \in \mathcal{V}$, and "." here and below refers to the standard Euclidean scalar product of the $n$-component vectors.

For $\boldsymbol{\gamma} \in \mathcal{V}^{*}$ define $[1,5,31]$ its Lie derivative along $\boldsymbol{Q} \in \mathcal{V}$ as $L_{\boldsymbol{Q}}(\boldsymbol{\gamma})=\boldsymbol{\gamma}^{\prime}[\boldsymbol{Q}]-\left(\boldsymbol{Q}^{\prime}\right)^{\dagger}(\boldsymbol{\gamma})$, see e.g. [5, 31] for further details.

For $\boldsymbol{Q} \in \mathcal{V}$ and $L=\sum_{j=-\infty}^{m} h_{j} D^{j}$ we set $L^{\prime}[\boldsymbol{Q}]=\sum_{j=-\infty}^{m} h_{j}^{\prime}[\boldsymbol{Q}] D^{j}$.

If $\boldsymbol{Q} \in \mathcal{V}$ and $\boldsymbol{\gamma} \in \mathcal{V}^{*}$ then we have [24] $\delta(\boldsymbol{Q} \cdot \boldsymbol{\gamma}) / \delta \boldsymbol{u}=\left(\boldsymbol{Q}^{\prime}\right)^{\dagger}(\boldsymbol{\gamma})+\left(\boldsymbol{\gamma}^{\prime}\right)^{\dagger}(\boldsymbol{Q})$. Hence if $\left(\boldsymbol{\gamma}^{\prime}\right)^{\dagger}(\boldsymbol{Q})-\boldsymbol{\gamma}^{\prime}[\boldsymbol{Q}]=0$ then we obtain $[27]$

$$
L_{\boldsymbol{Q}}(\gamma)=\delta(\boldsymbol{Q} \cdot \gamma) / \delta \boldsymbol{u}
$$

For weakly nonlocal $R: \mathcal{V} \rightarrow \mathcal{V}, J: \mathcal{V} \rightarrow \mathcal{V}^{*}, P: \mathcal{V}^{*} \rightarrow \mathcal{V}, N: \mathcal{V}^{*} \rightarrow \mathcal{V}^{*}$ define [12] their Lie derivatives along a $\boldsymbol{Q} \in \mathcal{V}$ as follows: $L_{\boldsymbol{Q}}(R)=R^{\prime}[\boldsymbol{Q}]-\left[\boldsymbol{Q}^{\prime}, R\right], L_{\boldsymbol{Q}}(N)=N^{\prime}[\boldsymbol{Q}]+\left[\boldsymbol{Q}^{\prime \dagger}, N\right]$, $L_{\boldsymbol{Q}}(P)=P^{\prime}[\boldsymbol{Q}]-P \circ \boldsymbol{Q}^{\prime}-\boldsymbol{Q}^{\prime \dagger} \circ P, L_{\boldsymbol{Q}}(J)=J^{\prime}[\boldsymbol{Q}]+J \circ \boldsymbol{Q}^{\prime}+\boldsymbol{Q}^{\prime \dagger} \circ J$. Here and below we do not assume $R$ and $J$ to be defined on the whole of $\mathcal{V}$, respectively $P$ and $N$ on the whole of $\mathcal{V}^{*}$.

We shall call an operator $J: \mathcal{V} \rightarrow \mathcal{V}^{*}$ (respectively $P: \mathcal{V}^{*} \rightarrow \mathcal{V}$ ) formally skew-symmetric if it is skew-symmetric when considered as a formal series, i.e., $J^{\dagger}=-J$ (respectively $P^{\dagger}=-P$ ).

Recall that the proper way to extend the concept of the finite-dimensional Hamiltonian structure to evolutionary systems of PDEs in $(1+1)$ dimensions is the following one. A formally skew-symmetric operator $P: \mathcal{V}^{*} \rightarrow \mathcal{V}$ is Hamiltonian [5] (or implectic [12]) if its Schouten bracket with itself vanishes: $[P, P]=0$. The Schouten bracket $[\cdot, \cdot]$ is given by the formula

$$
[H, K]\left(\chi_{1}, \chi_{2}, \chi_{3}\right)=\left\langle H L_{K \chi_{1}}\left(\chi_{2}\right), \chi_{3}\right\rangle+\left\langle K L_{H \chi_{1}}\left(\chi_{2}\right), \chi_{3}\right\rangle+\operatorname{cycle}(1,2,3),
$$

where $\chi_{i} \in \mathcal{V}^{*}$ and $\langle$,$\rangle is given by (2), see e.g. [5]. Throughout the rest of the paper [\cdot, \cdot]$ will denote the Schouten bracket rather than the commutator.

Two Hamiltonian operators are said to be compatible [12] (or to form a Hamiltonian pair [5]) if any linear combination thereof is again a Hamiltonian operator. Note that the Hamiltonian operators are compatible if and only if their Schouten bracket vanishes [5]. 
The Poisson bracket $\{,\}_{P}$ associated with a Hamiltonian operator $P$ is (see e.g. [5, 24]) a mapping from $\overline{\mathcal{A}} \times \overline{\mathcal{A}}$ to $\overline{\mathcal{A}}$ given by the formula

$$
\{\mathcal{F}, \mathcal{G}\}_{P}=\int d x \delta \mathcal{F} P(\delta \mathcal{G})
$$

for any $\mathcal{F}, \mathcal{G} \in \overline{\mathcal{A}}$. Here we set $\delta \mathcal{F} \stackrel{\text { def }}{=} \delta f / \delta \boldsymbol{u}$ for any $\mathcal{F}=\int f d x \in \overline{\mathcal{A}}$.

A formally skew-symmetric operator $J: \mathcal{V} \rightarrow \mathcal{V}^{*}$ is symplectic [12] if

$$
\left\langle J^{\prime}[\boldsymbol{P}] \boldsymbol{Q}, \boldsymbol{R}\right\rangle+\left\langle J^{\prime}[\boldsymbol{Q}] \boldsymbol{R}, \boldsymbol{P}\right\rangle+\left\langle J^{\prime}[\boldsymbol{R}] \boldsymbol{P}, \boldsymbol{Q}\right\rangle=0
$$

for any $\boldsymbol{P}, \boldsymbol{Q}, \boldsymbol{R} \in \mathcal{V}$.

Following the tradition established in the literature we shall sometimes speak of Hamiltonian (or symplectic) structures rather than of Hamiltonian (or symplectic) operators, even though the latter terms are equivalent with the former.

We shall call a Hamiltonian or symplectic operator nondegenerate if it is nondegenerate as a formal series in powers of $D$. A nondegenerate operator $P: \mathcal{V}^{*} \rightarrow \mathcal{V}$ is Hamiltonian if and only if $P^{-1}$ is symplectic. Following [12], and in contrast with a number of other references, in what follows we do not assume symplectic operators to be a priori nondegenerate.

We have the following homotopy formula (see [24, Ch. 5] and $[5,23]$ for details): if $J: \mathcal{V} \rightarrow \mathcal{V}^{*}$ is a differential symplectic operator and $M \times B$ is a star-shaped domain (recall that $M$ and $B$ are domains of values of $\boldsymbol{u}$ and $x$, respectively) then we have $J=\boldsymbol{\zeta}^{\prime}-\boldsymbol{\zeta}^{\prime \dagger}$ for

$$
\boldsymbol{\zeta}=\int_{0}^{1}(J(\boldsymbol{u}))[\lambda \boldsymbol{u}] d \lambda
$$

Here $J(\boldsymbol{u})$ means the result of action of the differential operator $J$ on the vector $\boldsymbol{u}$, and for any $f \in \mathcal{A}$ the quantity $f[\lambda \boldsymbol{u}]$ is defined as follows: if $f=f\left(x, \boldsymbol{u}, \ldots, \boldsymbol{u}_{k}\right)$ then

$$
f[\lambda \boldsymbol{u}] \stackrel{\text { def }}{=} f\left(x, \lambda \boldsymbol{u}, \ldots, \lambda \boldsymbol{u}_{k}\right) .
$$

In what follows we make the blanket assumption that $M \times B$ is a star-shaped domain so that (7) is automatically valid.

In order to see how (7) works, consider the following simple example. Let $J=D$. Then we have $J(\boldsymbol{u})=D(\boldsymbol{u})=\boldsymbol{u}_{1}$, and therefore $(J(\boldsymbol{u}))[\lambda \boldsymbol{u}]=\lambda \boldsymbol{u}_{1}$. By (7) we obtain $\boldsymbol{\zeta}=\boldsymbol{u}_{1} / 2$ and indeed the equality $J=\boldsymbol{\zeta}^{\prime}-\boldsymbol{\zeta}^{\prime \dagger}$ holds, as desired.

Note that the proper geometrical framework for the above results is provided by the formal calculus of variations, and we refer the interested reader to [2, 5, 24, 31] and references therein for further details.

Our immediate goal is to generalize (7) to the case when the matrix operator $J$ is weakly nonlocal rather than purely differential, see Theorem 2 below. However, we shall need a few more definitions and known results in order to proceed.

A symplectic operator $J$ is compatible [12] with a Hamiltonian operator $\tilde{P}$ if $J \tilde{P} J$ is again symplectic. If the symplectic operator $J$ is an inverse of a Hamiltonian operator $P$, then the compatibility of $J$ and $\tilde{P}$ is equivalent to that of $P$ and $\tilde{P}$. In fact, a more general assertion holds.

Lemma 1. Consider a nondegenerate Hamiltonian operator $P$ and a formally skew-symmetric operator $\tilde{P}: \mathcal{V}^{*} \rightarrow \mathcal{V}$ which is not necessarily Hamiltonian. Their Schouten bracket vanishes $([P, \tilde{P}]=0)$ if and only if the operator $P^{-1} \tilde{P} P^{-1}$ is symplectic.

Sketch of proof. By (6), the operator $\widetilde{J}=P^{-1} \tilde{P} P^{-1}$ is symplectic if and only if

$$
\left\langle\widetilde{J}^{\prime}\left[\boldsymbol{X}_{1}\right] \boldsymbol{X}_{2}, \boldsymbol{X}_{3}\right\rangle+\left\langle\widetilde{J}^{\prime}\left[\boldsymbol{X}_{2}\right] \boldsymbol{X}_{3}, \boldsymbol{X}_{1}\right\rangle+\left\langle\widetilde{J}^{\prime}\left[\boldsymbol{X}_{3}\right] \boldsymbol{X}_{1}, \boldsymbol{X}_{2}\right\rangle=0 .
$$


Let $\boldsymbol{X}_{i}=P \boldsymbol{\chi}_{i}, \boldsymbol{\chi}_{i} \in \mathcal{V}$. By equation (4.12) and Proposition 4.3 of [30] which are readily seen to be applicable in the infinite-dimensional case as well, we have

$$
[P, \tilde{P}]\left(\chi_{1}, \chi_{2}, \chi_{3}\right)=\left\langle\widetilde{J}^{\prime}\left[\boldsymbol{X}_{1}\right] \boldsymbol{X}_{2}, \boldsymbol{X}_{3}\right\rangle+\left\langle\widetilde{J}^{\prime}\left[\boldsymbol{X}_{2}\right] \boldsymbol{X}_{3}, \boldsymbol{X}_{1}\right\rangle+\left\langle\widetilde{J}^{\prime}\left[\boldsymbol{X}_{3}\right] \boldsymbol{X}_{1}, \boldsymbol{X}_{2}\right\rangle,
$$

and the result follows.

Note also the following easy corollary of Theorem 1 of [15].

Theorem 1. Let $\varepsilon_{\alpha}$ be arbitrary nonzero constants, and $\psi_{\alpha} \in \mathcal{A}$ be local functions such that $\delta \psi_{\alpha} / \delta \boldsymbol{u} \neq 0$ for all $\alpha=1, \ldots, q$. Then the operator

$$
J=\sum_{\alpha=1}^{q} \varepsilon_{\alpha} \frac{\delta \psi_{\alpha}}{\delta \boldsymbol{u}} \otimes D^{-1} \circ \frac{\delta \psi_{\alpha}}{\delta \boldsymbol{u}}
$$

is symplectic.

We now need to extend $\mathcal{A}, \mathcal{V}$ and $\mathcal{V}^{*}$ to include weakly nonlocal elements. First of all, a $q$ component vector function $\vec{f}$ is said to be weakly nonlocal if there exist a nonnegative integer $s$ and $\overrightarrow{f_{0}} \in \mathcal{A}^{q}, \vec{f}_{\alpha} \in \mathcal{A}^{q}, K_{\alpha} \in \mathcal{A}, \alpha=1, \ldots, s$ such that $\vec{f}$ can be written as

$$
\vec{f}=\overrightarrow{f_{0}}+\sum_{\alpha=1}^{s} \vec{f}_{\alpha} D^{-1}\left(K_{\alpha}\right)
$$

where $\vec{f}_{\alpha}$ are linearly independent over $\mathcal{A}$ for $\alpha=1, \ldots, s, \delta K_{\alpha} / \delta \boldsymbol{u} \neq 0, \alpha=1, \ldots, s$, and $K_{\alpha}$ are linearly independent over the constants.

We shall denote the space of weakly nonlocal $q$-component vectors in the sense of above definition by $\tilde{\mathcal{A}}^{q} ; \tilde{\mathcal{V}}$ (resp. $\tilde{\mathcal{V}}^{*}$ ) will stand for the space of $n$-component columns (resp. rows) with entries from $\tilde{\mathcal{A}} \equiv \tilde{\mathcal{A}}^{1}$. The definition of directional derivative is extended to $\tilde{\mathcal{A}}^{q}$ as follows: for $\vec{f}$ of the form (10) we set

$$
\vec{f}^{\prime}=\vec{f}_{0}^{\prime}+\sum_{\alpha=1}^{s}\left(D^{-1}\left(K_{\alpha}\right) \vec{f}_{\alpha}^{\prime}+\vec{f}_{\alpha} D^{-1} \circ K_{\alpha}^{\prime}\right) .
$$

Moreover, the definitions of directional derivative and the Lie derivative along $\boldsymbol{Q} \in \mathcal{V}$ readily extend to the elements of $\tilde{\mathcal{V}}$. In the present paper we adopt a relatively informal approach to nonlocal variables in spirit of [11]. For a more rigorous approach to nonlocal symmetries see e.g. $[2,25]$ and references therein.

We shall call a weakly nonlocal Hamiltonian operator $P$ normal if for any $\boldsymbol{Q} \in \tilde{\mathcal{V}}$ the condition $L_{\boldsymbol{Q}}(P)=0$ implies that $\boldsymbol{Q} \in \mathcal{V}$.

\section{Main results}

We start with the following nonlocal generalization of the homotopy formula (7).

Theorem 2. Let $J: \mathcal{V} \rightarrow \mathcal{V}^{*}$ be a weakly nonlocal formally skew-symmetric operator. Suppose that there exist $\varepsilon_{\alpha}$ and local $H_{\alpha}$ such that $\varepsilon_{\alpha}^{2}=1$ (i.e., $\varepsilon_{\alpha}= \pm 1$ ) and we have

$$
J_{-}=\sum_{\alpha=1}^{q} \varepsilon_{\alpha} \delta H_{\alpha} / \delta \boldsymbol{u} \otimes D^{-1} \circ \delta H_{\alpha} / \delta \boldsymbol{u} .
$$

Then the operator $J$ is symplectic if and only if there exists a local $\gamma_{0} \in \mathcal{V}^{*}$ such that we have $J=\gamma^{\prime}-\left(\gamma^{\prime}\right)^{\dagger}$ for

$$
\boldsymbol{\gamma}=\gamma_{0}+\frac{1}{2} \sum_{\alpha=1}^{q} \varepsilon_{\alpha} \delta H_{\alpha} / \delta \boldsymbol{u} D^{-1}\left(H_{\alpha}\right) .
$$


Proof. If there exists $\gamma_{0}$ such that $\gamma(11)$ satisfies

$$
J=\gamma^{\prime}-\left(\gamma^{\prime}\right)^{\dagger}
$$

then $J$ is obviously symplectic.

Now assume that $J$ is symplectic and construct a suitable $\gamma_{0}$ such that $\boldsymbol{\gamma}(11)$ satisfies (12). Let $\widetilde{\gamma}=\gamma-\gamma_{0}$. We readily see that we have

$$
\left(\widetilde{\gamma}^{\prime}-\widetilde{\gamma}^{\prime \dagger}\right)_{-}=J_{-}
$$

On the other hand, $\left(\widetilde{\gamma}^{\prime}-\widetilde{\gamma}^{\prime \dagger}\right)$ obviously is a symplectic operator and therefore so is

$$
\tilde{J}=J-\left(\widetilde{\gamma}^{\prime}-\widetilde{\gamma}^{\prime \dagger}\right) \text {. }
$$

By virtue of (13) we have $\tilde{J}_{-}=0$, i.e., $\tilde{J}$ is purely differential. Let

$$
\gamma_{0}=\int_{0}^{1}(\tilde{J}(\boldsymbol{u}))[\lambda \boldsymbol{u}] d \lambda
$$

Clearly, this $\gamma_{0}$ is local [5], and by (7) we have $\tilde{J}=\gamma_{0}^{\prime}-\left(\gamma_{0}^{\prime}\right)^{\dagger}$. Hence $\gamma(11)$ satisfies (12), and the result follows.

Theorem 2 means that the existence of a (not necessarily globally defined) weakly nonlocal $\gamma$ such that (12) holds is a necessary and sufficient condition for a weakly nonlocal $J$ to be symplectic. An important feature of this result is that the nonlocal terms in $\gamma$ are uniquely determined by the structure of nonlocal terms in $J$, so in fact we only need to determine a local $\gamma_{0}$.

Combining Lemma 1 and Theorem 2 we arrive at the following results.

Corollary 1. Let $P$ be a nondegenerate Hamiltonian operator and $\tilde{P}: \mathcal{V}^{*} \rightarrow \mathcal{V}$ be a formally skew-symmetric operator such that $P^{-1} \tilde{P} P^{-1}$ is weakly nonlocal and there exist $\varepsilon_{\alpha}= \pm 1$ and local $F_{\alpha}$ such that

$$
P^{-1} \tilde{P} P^{-1}=\sum_{\alpha=1}^{s} \varepsilon_{\alpha} \delta F_{\alpha} / \delta \boldsymbol{u} \otimes D^{-1} \circ \delta F_{\alpha} / \delta \boldsymbol{u} .
$$

Then $[P, \tilde{P}]=0$ if and only if there exists a local $\gamma_{0} \in \mathcal{V}^{*}$ such that

$$
\boldsymbol{\gamma}=\boldsymbol{\gamma}_{0}+\frac{1}{2} \sum_{\alpha=1}^{s} \varepsilon_{\alpha} \delta F_{\alpha} / \delta \boldsymbol{u} D^{-1}\left(F_{\alpha}\right)
$$

satisfies $P^{-1} \tilde{P} P^{-1}=\gamma^{\prime}-\left(\gamma^{\prime}\right)^{\dagger}$.

Corollary 2. Under the assumptions of Corollary 1 suppose that $P$ is a normal weakly nonlocal Hamiltonian operator of the form

$$
P=\sum_{m=0}^{\bar{p}} a_{m} D^{m}+\sum_{\rho=1}^{\bar{q}} \bar{\epsilon}_{\rho} \boldsymbol{G}_{\rho} \otimes D^{-1} \circ \boldsymbol{G}_{\rho},
$$

where $a_{m}$ are $n \times n$ matrices with entries from $\mathcal{A}, \bar{\epsilon}_{\rho}$ are arbitrary nonzero constants, $\boldsymbol{G}_{\rho} \in \mathcal{V}$, and we have

$$
L_{\boldsymbol{G}_{\rho}}\left(\delta F_{\alpha} / \delta \boldsymbol{u}\right)=0, \quad \alpha=1, \ldots, s, \quad \rho=1, \ldots, \bar{p} .
$$

Then $[P, \tilde{P}]=0$ if and only if there exists a weakly nonlocal $\boldsymbol{\tau} \in \tilde{\mathcal{V}}$ such that $\tilde{P}=L_{\boldsymbol{\tau}}(P)$. 
Proof. Under the assumptions of Corollary 1 let $\boldsymbol{\tau}=-P \boldsymbol{\gamma}+\boldsymbol{Q}$, where $\boldsymbol{\gamma}$ is given by (15) and $\boldsymbol{Q}$ satisfies $L_{\boldsymbol{Q}}(P)=0$. Then we have $\tilde{P}=L_{\boldsymbol{\tau}}(P)$, cf. proof of Proposition 3 in [26].

The Hamiltonian operator $P$ is normal by assumption, and hence $\boldsymbol{Q}$ is local, i.e., $\boldsymbol{Q} \in \mathcal{V}$. Hence the only nonlocal terms in $\tau$ originate from $-P \gamma$ and read

$$
-\frac{1}{2} \sum_{\alpha=1}^{s} \varepsilon_{\alpha} D^{-1}\left(F_{\alpha}\right) P\left(\delta F_{\alpha} / \delta \boldsymbol{u}\right)+\frac{1}{2} \sum_{\alpha=1}^{q} \sum_{\rho=1}^{\bar{q}} \varepsilon_{\alpha} \bar{\varepsilon}_{\rho} \boldsymbol{G}_{\rho} D^{-1}\left(\left(D^{-1}\left(\delta F_{\alpha} / \delta \boldsymbol{u} \cdot \boldsymbol{G}_{\rho}\right)\right) F_{\alpha}\right) .
$$

Now, the expressions $D^{-1}\left(\left(\delta F_{\alpha} / \delta \boldsymbol{u} \cdot \boldsymbol{G}_{\rho}\right)\right)$ are in fact local. Indeed, by (3) the conditions (17) are equivalent to

$$
\delta\left(\boldsymbol{G}_{\rho} \cdot \delta F_{\alpha} / \delta \boldsymbol{u}\right) / \delta \boldsymbol{u}=0, \quad \alpha=1, \ldots, q, \quad \rho=1, \ldots, \bar{q} .
$$

In turn, (18) implies that $\left(\boldsymbol{G}_{\rho} \cdot \delta F_{\alpha} / \delta \boldsymbol{u}\right) \in \operatorname{Im} D$, as desired.

Hence $P\left(\delta F_{\alpha} / \delta \boldsymbol{u}\right)$ and $\left(D^{-1}\left(\delta F_{\alpha} / \delta \boldsymbol{u} \cdot \boldsymbol{G}_{\rho}\right)\right) F_{\alpha}$ are local, and $\boldsymbol{\tau}$ is weakly nonlocal.

On the other hand, if there exists a weakly nonlocal $\boldsymbol{\tau}$ such that $\tilde{P}=L_{\boldsymbol{\tau}}(P)$ then we have $[P, \tilde{P}]=0$, cf. the proof of Proposition 7.8 of [5] or equation (4) of [26], and the result follows.

The above two results are more than a mere test of whether a given $\tilde{P}$ has a zero Schouten bracket with $P$ (and, in particular, whether the Hamiltonian operators $P$ and $\tilde{P}$ are compatible). In particular, Corollary 2 shows that if $P$ is purely differential and normal then, under certain technical assumptions that appear to hold in all interesting examples, all weakly nonlocal Hamiltonian operators compatible with $P$ can be written in the form $L_{\boldsymbol{\tau}}(P)$ for suitably chosen weakly nonlocal $\boldsymbol{\tau}$.

Therefore, we can search for Hamiltonian operators compatible with $P$ by picking a general weakly nonlocal $\boldsymbol{\tau}$ and requiring the operator $L_{\boldsymbol{\tau}}(P)$ to be Hamiltonian. Clearly, we have considerably fewer unknown functions to determine than if we would just assume that $\tilde{P}$ is weakly nonlocal and formally skew-symmetric and then require $\tilde{P}$ to be a Hamiltonian operator compatible with $P$.

It is natural to ask under which conditions the operator $P^{-1} \tilde{P} P^{-1}$ meets the requirements of Corollary 1. To this end consider first a weakly nonlocal operator of the form

$$
J=\sum_{m=1}^{p} b_{m} D^{m}+\sum_{\alpha=1}^{q} \varepsilon_{\alpha} \frac{\delta \psi_{\alpha}}{\delta \boldsymbol{u}} \otimes D^{-1} \circ \frac{\delta \psi_{\alpha}}{\delta \boldsymbol{u}},
$$

where $b_{m}$ are $n \times n$ matrices with entries from $\mathcal{A}, \varepsilon_{\alpha}$ are arbitrary nonzero constants, and $\psi_{\alpha} \in \mathcal{A}$ are local functions.

In what follows we assume without loss of generality that $\delta \psi_{\alpha} / \delta \boldsymbol{u}, \alpha=1, \ldots, q$, are linearly independent over the constants. We have the following well-known result.

Lemma 2. Let $J: \mathcal{V} \rightarrow \mathcal{V}^{*}$ be a nondegenerate operator of the form (19). If $P=J^{-1}$ is a purely differential operator then we have

$$
P\left(\frac{\delta \psi_{\alpha}}{\delta \boldsymbol{u}}\right)=0, \quad \alpha=1, \ldots, q
$$

In particular, if $J$ is symplectic then $\int \psi_{\alpha} d x$ are Casimir functionals for the bracket $\{,\}_{P}$.

Proof. We have

$$
J(0)=\sum_{\alpha=1}^{q} c_{\alpha} \varepsilon_{\alpha} \frac{\delta \psi_{\alpha}}{\delta \boldsymbol{u}}
$$


where $c_{\alpha}$ are arbitrary constants. Acting by $P=J^{-1}$ on the left- and right-hand side of this equation yields

$$
\sum_{\alpha=1}^{q} c_{\alpha} \varepsilon_{\alpha} P\left(\frac{\delta \psi_{\alpha}}{\delta \boldsymbol{u}}\right)=0
$$

and since $c_{\alpha}$ are arbitrary we obtain (20).

Further let $\tilde{P}$ be a weakly nonlocal formally skew-symmetric operator of the form

$$
\tilde{P}=\sum_{m=0}^{\tilde{p}} \tilde{a}_{m} D^{m}+\sum_{\rho=1}^{\tilde{q}} \tilde{\varepsilon}_{\rho} \boldsymbol{Y}_{\rho} \otimes D^{-1} \circ \boldsymbol{Y}_{\rho}
$$

where $\tilde{a}_{m}$ are $n \times n$ matrices with entries from $\mathcal{A}$ and $\tilde{\varepsilon}_{\rho}$ are arbitrary nonzero constants.

Theorem 3. Let $J$ be a weakly nonlocal symplectic operator of the form (19) and $\tilde{P}: \mathcal{V}^{*} \rightarrow \mathcal{V}$ be a weakly nonlocal formally skew-symmetric operator of the form (21). Suppose that there exist local functions $H_{\rho}$ and $K_{\alpha}$ such that

$$
\begin{aligned}
& J \boldsymbol{Y}_{\rho}=\delta H_{\rho} / \delta \boldsymbol{u}, \quad \rho=1, \ldots, \tilde{q}, \quad \text { and } \\
& J \tilde{P}\left(\delta \psi_{\alpha} / \delta \boldsymbol{u}\right)=\delta K_{\alpha} / \delta \boldsymbol{u}, \quad \alpha=1, \ldots, q,
\end{aligned}
$$

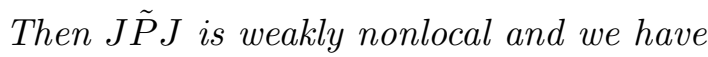

$$
\begin{aligned}
(J \tilde{P} J)_{-}= & \sum_{\alpha=1}^{q} \varepsilon_{\alpha}\left(\frac{\delta K_{\alpha}}{\delta \boldsymbol{u}} \otimes D^{-1} \circ \frac{\delta \psi_{\alpha}}{\delta \boldsymbol{u}}+\frac{\delta \psi_{\alpha}}{\delta \boldsymbol{u}} \otimes D^{-1} \circ \frac{\delta K_{\alpha}}{\delta \boldsymbol{u}}\right) \\
& -\sum_{\rho=1}^{\tilde{q}} \tilde{\epsilon}_{\rho} \frac{\delta H_{\rho}}{\delta \boldsymbol{u}} \otimes D^{-1} \circ \frac{\delta H_{\rho}}{\delta \boldsymbol{u}} .
\end{aligned}
$$

Moreover, the operator $J \tilde{P} J$ is symplectic if and only if there exists a local $\gamma_{0} \in \mathcal{V}^{*}$ such that

$$
\boldsymbol{\gamma}=\gamma_{0}-\frac{1}{2} \sum_{\rho=1}^{\tilde{q}} \tilde{\epsilon}_{\rho} \frac{\delta H_{\rho}}{\delta \boldsymbol{u}} D^{-1}\left(H_{\rho}\right)+\frac{1}{2} \sum_{\alpha=1}^{q} \varepsilon_{\alpha}\left(\frac{\delta K_{\alpha}}{\delta \boldsymbol{u}} D^{-1}\left(\psi_{\alpha}\right)+\frac{\delta \psi_{\alpha}}{\delta \boldsymbol{u}} D^{-1}\left(K_{\alpha}\right)\right)
$$

satisfies $J \tilde{P} J=\gamma^{\prime}-\left(\gamma^{\prime}\right)^{\dagger}$.

The proof is by straightforward computation. Note that imposing the conditions (22) is a very weak restriction, as (22) can be shown to follow from weak nonlocality and symplecticity of $J \tilde{P} J$ under certain minor technical assumptions.

The conditions (22) have a very simple meaning. The first of these conditions ensures that $L_{\boldsymbol{Y}_{\rho}}(J)=0$, i.e., $\boldsymbol{Y}_{\rho}$ are Hamiltonian with respect to $J$. The second condition means that the action of the operator $N=J \tilde{P}$ on $\delta \psi_{\alpha} / \delta \boldsymbol{u}$ yields a variational derivative of another Hamiltonian density $K_{\alpha}$. Moreover, if the operator $N^{\dagger}=\tilde{P} J$ is hereditary, the said second condition guarantees [23] that $N^{k}\left(\delta \psi_{\alpha} / \delta \boldsymbol{u}\right)$ are variational derivatives (of possibly nonlocal Hamiltonian densities) for all $k=2,3, \ldots$.

Combining Theorem 3 and Corollary 2 we readily obtain the following results.

Corollary 3. Let $P$ be a nondegenerate Hamiltonian operator such that $J=P^{-1}$ is weakly nonlocal and can be written in the form (19) for suitable $p, q, b_{m}$ and $\psi_{\alpha}$. Then under the assumptions of Theorem 3 any formally skew-symmetric operator $\tilde{P}: \mathcal{V}^{*} \rightarrow \mathcal{V}$ such that $[P, \tilde{P}]=0$ can be written as $\tilde{P}=L_{\boldsymbol{\tau}}(P)$, where $\boldsymbol{\tau}=-P \boldsymbol{\gamma}$ and $\boldsymbol{\gamma}$ is given by (24). 
Corollary 4. Under the assumptions of Corollary 3 suppose that $P$ is a weakly nonlocal operator of the form (16) and we have

$$
L_{\boldsymbol{G}_{\rho}}\left(\delta K_{\alpha} / \delta \boldsymbol{u}\right)=0, \quad L_{\boldsymbol{G}_{\rho}}\left(\delta \psi_{\alpha} / \delta \boldsymbol{u}\right)=0, \quad \alpha=1, \ldots, q, \quad \rho=1, \ldots, \bar{q} .
$$

Then $\boldsymbol{\tau}=-P \boldsymbol{\gamma}$ is weakly nonlocal.

Moreover, if $P$ is a differential operator then $\boldsymbol{\tau}=-P \gamma$ has the form

$$
\boldsymbol{\tau}=\boldsymbol{\tau}_{0}+\frac{1}{2} \sum_{\rho=1}^{\tilde{q}} \tilde{\epsilon}_{\rho} P\left(\frac{\delta H_{\rho}}{\delta \boldsymbol{u}}\right) D^{-1}\left(H_{\rho}\right)-\frac{1}{2} \sum_{\alpha=1}^{q} \varepsilon_{\alpha} P\left(\frac{\delta K_{\alpha}}{\delta \boldsymbol{u}}\right) D^{-1}\left(\psi_{\alpha}\right),
$$

where $\boldsymbol{\tau}_{0} \in \mathcal{V}$ is local.

Proof. Using (25) and Corollary 2 we readily see that under the assumptions made $\boldsymbol{\tau}=-P \boldsymbol{\gamma}$ is indeed weakly nonlocal. If $P$ is a differential operator then we have $P\left(\frac{\delta \psi_{\alpha}}{\delta \boldsymbol{u}}\right)=0$ by Lemma 2 , and a straightforward computation yields (26).

For instance, let $n=2$, and $\boldsymbol{u}=(u, v)^{T}$. Consider

$$
J=\left(\begin{array}{cc}
0 & 1 \\
-1 & 0
\end{array}\right) \quad \text { and } \quad \tilde{P}=\left(\begin{array}{cc}
D+2 v D^{-1} \circ v & -2 v D^{-1} \circ u \\
-2 u D^{-1} \circ v & D+2 u D^{-1} \circ u
\end{array}\right)
$$

the symplectic structure and the Hamiltonian structure for the nonlinear Schrödinger equation, see e.g. [32] and references therein. We can rewrite $\tilde{P}$ as

$$
\tilde{P}=\left(\begin{array}{cc}
D & 0 \\
0 & D
\end{array}\right)+\boldsymbol{Y}_{1} \otimes D^{-1} \circ \boldsymbol{Y}_{1}, \quad \boldsymbol{Y}_{1}=\sqrt{2}\left(\begin{array}{c}
-v \\
u
\end{array}\right) .
$$

We have

$$
\begin{aligned}
& J \tilde{P} J=\left(\begin{array}{cc}
-D-2 u D^{-1} \circ u & -2 u D^{-1} \circ v \\
-2 v D^{-1} \circ u & -D-2 v D^{-1} \circ v
\end{array}\right)=\left(\begin{array}{cc}
-D & 0 \\
0 & -D
\end{array}\right)-\frac{\delta H_{1}}{\delta \boldsymbol{u}} \otimes D^{-1} \circ \frac{\delta H_{1}}{\delta \boldsymbol{u}}, \\
& H_{1}=\left(u^{2}+v^{2}\right) / \sqrt{2} .
\end{aligned}
$$

The conditions of Theorem 2 and Corollary 3 are readily seen to hold, and therefore we have $J \tilde{P} J=\gamma^{\prime}-\left(\gamma^{\prime}\right)^{\dagger}$, where

$$
\boldsymbol{\gamma}=\gamma_{0}-\frac{1}{2} \frac{\delta H_{1}}{\delta \boldsymbol{u}} D^{-1}\left(H_{1}\right), \quad \gamma_{0}=\left(v_{1} / 2, u_{1} / 2\right),
$$

and $\tilde{P}=L_{\boldsymbol{\tau}}\left(J^{-1}\right)$, where

$$
\boldsymbol{\tau}=-\boldsymbol{u}_{1} / 2+\frac{1}{2} \boldsymbol{Y}_{1} D^{-1}\left(H_{1}\right)
$$

Given a Hamiltonian operator $P$, it is natural to ask under which conditions $\tilde{P}=L_{\boldsymbol{\tau}}(P)$ also is a Hamiltonian operator. A straightforward but tedious computation yields the following

Theorem 4. Under the assumptions of Corollary 3 suppose that there exist local functions $L_{\rho}$ and $M_{\alpha}$ such that

$$
\begin{aligned}
& J \tilde{P}\left(\delta H_{\rho} / \delta \boldsymbol{u}\right)=\delta L_{\rho} / \delta \boldsymbol{u}, \quad \rho=1, \ldots, \tilde{q}, \quad \text { and } \\
& J \tilde{P}\left(\delta K_{\alpha} / \delta \boldsymbol{u}\right)=\delta M_{\alpha} / \delta \boldsymbol{u}, \quad \alpha=1, \ldots, q .
\end{aligned}
$$


Then $\tilde{P}=L_{\boldsymbol{\tau}}(P)$ is a Hamiltonian operator if and only if there exists a local $\widetilde{\gamma}_{0} \in \mathcal{V}^{*}$ such that

$$
\begin{aligned}
\widetilde{\boldsymbol{\gamma}}= & \widetilde{\boldsymbol{\gamma}}_{0}-\frac{1}{2} \sum_{\rho=1}^{\tilde{q}} \tilde{\epsilon}_{\rho}\left(\frac{\delta L_{\rho}}{\delta \boldsymbol{u}} D^{-1}\left(H_{\rho}\right)+\frac{\delta H_{\rho}}{\delta \boldsymbol{u}} D^{-1}\left(L_{\rho}\right)\right) \\
& +\frac{1}{2} \sum_{\alpha=1}^{q} \varepsilon_{\alpha}\left(\frac{\delta M_{\alpha}}{\delta \boldsymbol{u}} D^{-1}\left(\psi_{\alpha}\right)+\frac{\delta K_{\alpha}}{\delta \boldsymbol{u}} D^{-1}\left(K_{\alpha}\right)+\frac{\delta \psi_{\alpha}}{\delta \boldsymbol{u}} D^{-1}\left(M_{\alpha}\right)\right)
\end{aligned}
$$

satisfies $(J \tilde{P})^{2} J=\tilde{\gamma}^{\prime}-\left(\tilde{\gamma}^{\prime}\right)^{\dagger}$.

Proof. By Proposition 1 of $[26]$ the operator $\tilde{P}=L_{\boldsymbol{\tau}}(P)$ is Hamiltonian if and only if

$$
\left[L_{\boldsymbol{\tau}}^{2}(P), P\right]=0
$$

If $P$ is nondegenerate then by Lemma 1 the condition (29) is equivalent to the requirement that the operator $J L_{\boldsymbol{\tau}}^{2}(P) J$ be symplectic. It is readily seen that

$$
J L_{\boldsymbol{\tau}}^{2}(P) J=J L_{\boldsymbol{\tau}}(\tilde{P}) J=L_{\boldsymbol{\tau}}(J \tilde{P} J)+2(J \tilde{P})^{2} J
$$

In turn, as $J \tilde{P} J$ is symplectic, we have $L_{\boldsymbol{\tau}}(J \tilde{P} J)=(J \tilde{P} J \boldsymbol{\tau})^{\prime}-(J \tilde{P} J \boldsymbol{\tau})^{\prime \dagger}$, and, as $\boldsymbol{\tau}=-P \boldsymbol{\gamma}=$ $-J^{-1} \gamma$, where $\boldsymbol{\gamma}$ is given by (24), we obtain

$$
L_{\boldsymbol{\tau}}(J \tilde{P} J)=-(J \tilde{P} \gamma)^{\prime}+(J \tilde{P} \gamma)^{\prime \dagger},
$$

so the operator $L_{\boldsymbol{\tau}}(J \tilde{P} J)$ is symplectic.

Hence the operator $J L_{\boldsymbol{\tau}}^{2}(P) J$ is symplectic if and only if so is $(J \tilde{P})^{2} J$. By virtue of $(27)$ the operator $(J \tilde{P})^{2} J$ is weakly nonlocal, so we can verify its symplecticity using Theorem 2 , and the result follows.

Combining Theorem 4 and Corollary 2 we obtain the following

Corollary 5. Under the assumptions of Theorem 4 suppose that $P$ is normal, weakly nonlocal and has the form (16). Further assume that we have

$$
\begin{array}{llll}
L_{\boldsymbol{G}_{\rho}}\left(\delta H_{\sigma} / \delta \boldsymbol{u}\right)=0, & L_{\boldsymbol{G}_{\rho}}\left(\delta L_{\sigma} / \delta \boldsymbol{u}\right)=0, & \rho=1, \ldots, \bar{q}, & \sigma=1, \ldots, \tilde{q}, \\
L_{\boldsymbol{G}_{\rho}}\left(\delta K_{\beta} / \delta \boldsymbol{u}\right)=0, & L_{\boldsymbol{G}_{\rho}}\left(\delta M_{\beta} / \delta \boldsymbol{u}\right)=0, & & \\
& L_{\boldsymbol{G}_{\rho}}\left(\delta \psi_{\beta} / \delta \boldsymbol{u}\right)=0, & \rho=1, \ldots, \bar{q}, & \beta=1, \ldots, q, \\
L_{\boldsymbol{Y}_{\rho}}\left(\delta H_{\sigma} / \delta \boldsymbol{u}\right)=0, & L_{\boldsymbol{Y}_{\rho}}\left(\delta K_{\alpha} / \delta \boldsymbol{u}\right)=0, & & \\
& L_{\boldsymbol{Y}_{\rho}}\left(\delta \psi_{\alpha} / \delta \boldsymbol{u}\right)=0, & \alpha=1, \ldots, q, & \rho, \sigma=1, \ldots, \tilde{q},
\end{array}
$$

Then $\tilde{P}$ is a Hamiltonian operator if and only if there exists a weakly nonlocal $\tilde{\boldsymbol{\tau}} \in \tilde{\mathcal{V}}$ such that $L_{\boldsymbol{\tau}}^{2}(P)=L_{\tilde{\boldsymbol{\tau}}}(P)$.

Proof. We readily find that $\tilde{\boldsymbol{\tau}}=-P(-J \tilde{P} \boldsymbol{\gamma}+2 \tilde{\boldsymbol{\gamma}})+\boldsymbol{Q}=\tilde{P} \boldsymbol{\gamma}-2 P \tilde{\boldsymbol{\gamma}}+\boldsymbol{Q}$, where $\boldsymbol{Q} \in \mathcal{V}$ because $P$ is normal. In complete analogy with the proof of Corollary 2 we find that the conditions (30) ensure that the coefficients at the nonlocal variables in $\tilde{\boldsymbol{\tau}}$ are local, and therefore $\tilde{\boldsymbol{\tau}}$ is weakly nonlocal. 


\section{Local Hamiltonian operators of zero order}

Now assume that $J$ has the form

$$
J=b_{0}
$$

where $b_{0}$ is an $n \times n$ matrix with entries from $\mathcal{A}$.

A complete description of all symplectic operators of this form can be found in [20]. Namely, if $J(31)$ is symplectic then we have [20]

$$
b_{0}=\sum_{s=1}^{n} b_{0}^{(1, s)}(x, \boldsymbol{u}) u_{1}^{s}+b_{0}^{(0)}(x, \boldsymbol{u}),
$$

i.e., $b_{0}$ depends only on $x, \boldsymbol{u}, \boldsymbol{u}_{1}$ and, moreover, is linear in $\boldsymbol{u}_{1}$. Of course, for $J$ (31) to be symplectic the quantities $b_{0}^{(1, s)}$ and $b_{0}^{(0)}$ must satisfy certain further conditions, see [20] for details.

Corollary 6. Let $P$ be a nondegenerate Hamiltonian operator such that $J=P^{-1}$ has the form (31). Then any formally skew-symmetric differential operator $\tilde{P}: \mathcal{V}^{*} \rightarrow \mathcal{V}$ such that $[P, \tilde{P}]=0$ can be written as $\tilde{P}=L_{\boldsymbol{\tau}}(P)$ for a local $\boldsymbol{\tau} \in \mathcal{V}$.

Proof. Indeed, by Corollary 3 we can take $\boldsymbol{\tau}=-P \boldsymbol{\gamma}$ and $\boldsymbol{\gamma}$ given by (24) is now local.

Theorem 5. Let $P$ be a nondegenerate Hamiltonian operator such that $J=P^{-1}$ has the form (31). Then a formally skew-symmetric differential operator $\tilde{P}: \mathcal{V}^{*} \rightarrow \mathcal{V}$ is a Hamiltonian differential operator $\tilde{P}: \mathcal{V}^{*} \rightarrow \mathcal{V}$ compatible with $P$ if and only if there exist a local $\boldsymbol{\tau} \in \mathcal{V}$ and a local $\tilde{\boldsymbol{\tau}} \in \mathcal{V}$ such that $\tilde{P}=L_{\boldsymbol{\tau}}(P)$ and $L_{\boldsymbol{\tau}}^{2}(P)=L_{\tilde{\boldsymbol{\tau}}}(P)$.

Proof. The existence of a local $\boldsymbol{\tau} \in \mathcal{V}$ such that $\tilde{P}=L_{\boldsymbol{\tau}}(P)$ is immediate from Corollary 6 .

By Proposition 1 of [26] the operator $\tilde{P}=L_{\boldsymbol{\tau}}(P)$ is Hamiltonian if and only if $\left[L_{\boldsymbol{\tau}}^{2}(P), P\right]=0$. But by Corollary 6 the latter equality holds if and only if there exists a local $\tilde{\tau} \in \mathcal{V}$ such that $L_{\boldsymbol{\tau}}^{2}(P)=L_{\tilde{\boldsymbol{\tau}}}(P)$, and the result follows.

\section{Local Hamiltonian operators of Dubrovin-Novikov type}

Assume now that $P$ is a Hamiltonian operator of Dubrovin-Novikov type [6, 7], cf. also [8, 9, 10], i.e., it is a matrix differential operator with the entries

$$
P^{i j}=g^{i j}(\boldsymbol{u}) D+\sum_{k=1}^{n} b_{k}^{i j}(\boldsymbol{u}) u_{1}^{k},
$$

and $\operatorname{det} g^{i j} \neq 0$, i.e., $P$, considered as formal series, is nondegenerate.

An operator $P(33)$ with $\operatorname{det} g^{i j} \neq 0$ is $[6,7]$ a Hamiltonian operator if and only if $g^{i j}$ is a contravariant flat (pseudo-)Riemannian metric on an $n$-dimensional manifold $M$ with local coordinates $u^{i}$ and $b_{k}^{i j}=-\sum_{m=1}^{n} g^{i m} \Gamma_{m k}^{j}$, where $\Gamma_{m k}^{j}$ is the Levi-Civita connection associated with $g^{i j}: \Gamma_{i j}^{k}=(1 / 2) \sum_{s=1}^{n} g^{k s}\left(\partial g_{s j} / \partial x^{i}+\partial g_{i s} / \partial x^{j}-\partial g_{i j} / \partial x^{s}\right)$. Here $g_{i j}$ is determined from the conditions $\sum_{s=1}^{n} g^{k s} g_{s m}=\delta_{m}^{k}, k, m=1, \ldots, n$.

Let us pass to the flat coordinates $\psi^{\alpha}(\boldsymbol{u}), \alpha=1, \ldots, n$, of $g_{i j}$. In these coordinates $g^{i j}$ becomes a constant matrix $\eta^{i j}$, where $\eta^{i j}=0$ for $i \neq j$ and $\eta^{i i}$ satisfy $\left(\eta^{i i}\right)^{2}=1, i, j=1, \ldots, n$, and the Hamiltonian operator $P$ of Dubrovin-Novikov type associated with $g^{i j}$ takes the form

$$
P_{\mathrm{can}}^{i j}=\eta^{i j} D
$$


Theorem 6 ([4]). Let $P$ be a nondegenerate Hamiltonian operator of Dubrovin-Novikov type and $\tilde{P}: \mathcal{V}^{*} \rightarrow \mathcal{V}$ be a purely differential formally skew-symmetric operator such that

$$
\left\{\int \psi^{\alpha} d x, \int \psi^{\beta} d x\right\}_{\tilde{P}}=0, \quad \alpha, \beta=1, \ldots, n,
$$

where $\psi^{\alpha}=\psi^{\alpha}(\boldsymbol{u})$ are flat coordinates for the metric $g_{i j}$ associated with $P$. Then $[P, \tilde{P}]=0$ if and only if there exist a local $\boldsymbol{\tau} \in \mathcal{V}$ such that $\tilde{P}=L_{\boldsymbol{\tau}}(P)$.

Corollary 7. Under the assumptions of Theorem 6 suppose that

$$
\left\{\int \psi^{\alpha} d x, \int \psi^{\beta} d x\right\}_{L_{\tau}^{2}(P)}=0, \quad \alpha, \beta=1, \ldots, n,
$$

Then $\tilde{P}$ is a Hamiltonian operator compatible with $P$ if and only if there exists a local $\tilde{\boldsymbol{\tau}} \in \mathcal{V}$ such that $L_{\boldsymbol{\tau}}^{2}(P)=L_{\tilde{\boldsymbol{\tau}}}(P)$.

Proof. If there exist local $\boldsymbol{\tau}, \tilde{\boldsymbol{\tau}} \in \mathcal{V}$ such that $\tilde{P}=L_{\boldsymbol{\tau}}(P)$ and $L_{\boldsymbol{\tau}}^{2}(P)=L_{\tilde{\boldsymbol{\tau}}}(P)$ then by Proposition 3 of [26] the operator $\tilde{P}$ indeed is a Hamiltonian operator compatible with $P$. On the other hand, the existence of $\boldsymbol{\tau}$ such that $\tilde{P}=L_{\boldsymbol{\tau}}(P)$ is guaranteed by Theorem 6 . Thus we only have to show that if the operator $\tilde{P}$ is Hamiltonian then there exists a local $\tilde{\tau} \in \mathcal{V}$ such that $L_{\boldsymbol{\tau}}^{2}(P)=L_{\tilde{\boldsymbol{\tau}}}(P)$.

By Proposition 1 of $[26]$ the operator $\tilde{P}=L_{\boldsymbol{\tau}}(P)$ is Hamiltonian if and only if $\left[L_{\boldsymbol{\tau}}^{2}(P), P\right]=0$. As (36) holds by assumption, by Theorem 6 we have $\left[L_{\boldsymbol{\tau}}^{2}(P), P\right]=0$ if and only if there exists a local $\tilde{\boldsymbol{\tau}} \in \mathcal{V}$ such that $L_{\boldsymbol{\tau}}^{2}(P)=L_{\tilde{\boldsymbol{\tau}}}(P)$, and the result follows.

For a simple example, let $n=1, \boldsymbol{u} \equiv u$, and let $P=D$ and $\tilde{P}=D^{3}+2 u D+u_{1}$ be the first and the second Hamiltonian structure of the $\mathrm{KdV}$ equation. We have [4] $\tilde{P}=L_{\boldsymbol{\tau}}(P)$ for $\boldsymbol{\tau}=-\left(u^{2}+u_{2}\right) / 2$, and it is readily seen that the conditions of Corollary 7 are satisfied, so there exists a local $\tilde{\boldsymbol{\tau}}$ such that $L_{\boldsymbol{\tau}}^{2}(P)=L_{\tilde{\boldsymbol{\tau}}}(P)$. An easy computation shows that the latter equality holds e.g. for $\tilde{\boldsymbol{\tau}}=-u_{4} / 2-u_{1}^{2} / 2+5 u^{3} / 6$.

\section{Conclusions}

In the present paper we extended the homotopy formula (7) to a large class of weakly nonlocal symplectic structures, see Theorem 2 above. Besides the potential applications to the construction of nonlocal extensions for the variational complex, this result enabled us to provide a complete description for a large class of weakly nonlocal Hamiltonian operators compatible with a given nondegenerate weakly nonlocal Hamiltonian operator $P$ that possesses a weakly nonlocal inverse (Corollaries 2, 3, 4, and 5) or, more broadly, with a given weakly nonlocal symplectic operator $J$ (Theorems 3 and 4). These results admit useful simplifications for the case of zero- and first-order differential Hamiltonian operators, as presented in Sections 4 and 5. In particular, in Section 5 we provide a simple description for a very large class of local higher-order Hamiltonian operators compatible with a given local Hamiltonian operator of Dubrovin-Novikov type. Note that finding an efficient complete description of the nondegenerate weakly nonlocal Hamiltonian operators with a weakly nonlocal inverse is an interesting open problem, because such operators would naturally generalize the Hamiltonian operators (33) of Dubrovin-Novikov type from Section 5 and the zero-order local Hamiltonian operators from Section 4.

Thus, we extended the Lie derivative approach to the study of Hamiltonian operators compatible with a given Hamiltonian operator $P$ from finite-dimensional Poisson structures [26, 28] and Hamiltonian operators of Dubrovin-Novikov type [21, 26] to the weakly nonlocal Hamiltonian operators of more general form. An important advantage of this approach is that the 
vector fields $\boldsymbol{\tau}$ and $\tilde{\boldsymbol{\tau}}$ in general involve a considerably smaller number of unknown functions than a generic formally skew-symmetric operator being a "candidate" for a Hamiltonian operator compatible with $P$, and the search for such vector fields is often much easier than calculating directly the Schouten brackets involved, cf. also the discussion in [26, 28]. This could be very helpful in solving the classification problems like the following one: to describe all weakly nonlocal Hamiltonian operators compatible with a given Hamiltonian operator $P$ and having a certain prescribed form.

\section{Acknowledgements}

I am sincerely grateful to Prof. M. Błaszak and Drs. M. Marvan, E.V. Ferapontov, M.V. Pavlov and R.G. Smirnov for stimulating discussions. I am also pleased to thank the referees for useful suggestions.

This research was supported in part by the Czech Grant Agency (GA ČR) under grant No. 201/04/0538, by the Ministry of Education, Youth and Sports of the Czech Republic (MŠMT ČR) under grant MSM 4781305904 and by Silesian University in Opava under grant IGS 1/2004.

\section{References}

[1] Błaszak M., Multi-Hamiltonian theory of dynamical systems, Springer, Heidelberg, 1998.

[2] Bocharov A.V. et al., Symmetries and conservation laws for differential equations of mathematical physics, American Mathematical Society, Providence, RI, 1999.

[3] Cooke D.B., Compatibility conditions for Hamiltonian pairs, J. Math. Phys. 32 (1991), no. 11, 3071-3076.

[4] Degiovanni L., Magri F., Sciacca V., On deformation of Poisson manifolds of hydrodynamic type, Comm. Math. Phys. 253 (2005), 1-24, nlin.SI/0103052.

[5] Dorfman I., Dirac structures and integrability of nonlinear evolution equations, John Wiley \& Sons, Chichester, 1993.

[6] Dubrovin B.A., Novikov S.P., Hamiltonian formalism of one-dimensional systems of the hydrodynamic type and the Bogolyubov-Whitham averaging method, Soviet Math. Dokl. 27 (1983), 665-669.

[7] Dubrovin B.A., Novikov S.P., On Poisson brackets of hydrodynamic type, Soviet Math. Dokl. 30 (1984), 651-654.

[8] Ferapontov E.V., Compatible Poisson brackets of hydrodynamic type, J. Phys. A: Math. Gen. 34 (2001), 2377-2388, math.DG/0005221.

[9] Ferapontov E.V., Differential geometry of nonlocal Hamiltonian operators of hydrodynamic type, Funct. Anal. Appl. 25 (1991), 195-204.

[10] Ferapontov E.V., Nonlocal Hamiltonian operators of hydrodynamic type, differential geometry and applications, Am. Math. Soc. Trans. 170 (1995), 33-58.

[11] Finkel F., Fokas A.S., On the construction of evolution equations admitting a master symmetry, Phys. Lett. A 293 (2002), 36-44, nlin.SI/0112002.

[12] Fuchssteiner B., Fokas A.S., Symplectic structures, their Bäcklund transformations and hereditary symmetries, Phys. D 4 (1981/82), no. 1, 47-66.

[13] Lichnerowicz A., Les variétés de Poisson et leurs algèbres de Lie associées, J. Differential Geometry 12 (1977), no. 2, 253-300.

[14] Magri F., A simple model of the integrable Hamiltonian equation, J. Math. Phys. 19 (1978), 1156-1162.

[15] Maltsev A.Ya., Weakly nonlocal symplectic structures, Whitham method and weakly nonlocal symplectic structures of hydrodynamic type, J. Phys. A: Math. Gen. 38 (2005), 637-682, nlin.SI/0405060.

[16] Maltsev A.Ya., Novikov S.P., On the local systems Hamiltonian in the weakly non-local Poisson brackets, Phys. D 156 (2001), no. 1-2, 53-80, nlin.SI/0006030.

[17] Mikhailov A.V., Shabat A.B., Sokolov V.V., The symmetry approach to classification of integrable equations, in What is Integrability?, Editor V.E. Zakharov, Springer, New York, 1991, 115-184. 
[18] Mikhailov A.V., Shabat A.B., Yamilov R.I., The symmetry approach to classification of nonlinear equations. Complete lists of integrable systems, Russ. Math. Surv. 42 (1987), no. 4, 1-63.

[19] Mikhailov A.V., Yamilov R.I., Towards classification of (2+1)-dimensional integrable equations. Integrability conditions. I, J. Phys. A: Math. Gen. 31 (1998), 6707-6715.

[20] Mokhov O.I., Symplectic and Poisson geometry on loop sapces of manifolds in nonlinear equations, in Topics in Topology and Mathematical Physics, Editor S.P. Novikov, AMS, Providence, RI, 1995, 121-151, hep-th/9503076.

[21] Mokhov O.I., Compatible Dubrovin-Novikov Hamiltonian operators, Lie derivative and integrable systems of hydrodynamic type, Theoret. and Math. Phys. 133 (2002), no. 2, 1557-1564, math.DG/0201281.

[22] Mokhov O.I., Compatible nonlocal Poisson brackets of hydrodynamic type and related integrable hierarchies, Theoret. and Math. Phys. 132 (2002), no. 1, 942-954, math.DG/0201242.

[23] Oevel W., Rekursionmechanismen für Symmetrien und Erhaltungssätze in Integrablen Systemen, Ph.D. Thesis, University of Paderborn, Paderborn, 1984.

[24] Olver P.J., Applications of Lie groups to differential equations, Springer, New York, 1993.

[25] Sergyeyev A., On recursion operators and nonlocal symmetries of evolution equations, in Proc. Sem. Diff. Geom., Editor D. Krupka, Silesian University in Opava, Opava, 2000, 159-173, nlin.SI/0012011.

[26] Sergyeyev A., A simple way to make a Hamiltonian system into bi-Hamiltonian one, Acta Appl. Math. 83 (2004), 183-197, nlin.SI/0310012.

[27] Sergyeyev A., Why nonlocal recursion operators produce local symmetries: new results and applications, J. Phys. A: Math. Gen. 38 (2005), no. 15, 3397-3407, nlin.SI/0410049.

[28] Smirnov R.G., Bi-Hamiltonian formalism: a constructive approach, Lett. Math. Phys. 41 (1997), 333-347.

[29] Sokolov V.V., On symmetries of evolution equations, Russ. Math. Surv. 43 (1988), no. 5, 165-204.

[30] Vaisman I., Lectures on the geometry of Poisson manifolds, Birkhäuser, Basel, 1994.

[31] Wang J.P., Symmetries and conservation laws of evolution equations, Ph.D. Thesis, Vrije Universiteit van Amsterdam, Amsterdam, 1998.

[32] Wang J.P., A list of $1+1$ dimensional integrable equations and their properties, J. Nonlinear Math. Phys. 9 (2002), suppl. 1, 213-233. 